\title{
BMJ Open Use of glucose for pain management in premature neonates: a systematic review and meta-analysis protocol
}

Débora Joyce Duarte Oliveira, ${ }^{1}$ Kleyton Santos Medeiros, ${ }^{1}$ Ayane Cristine Alves Sarmento, ${ }^{1}$ Francisca Jennifer Duarte Oliveira, ${ }^{1}$ Ana Paula Ferreira Costa, ${ }^{1}$ Nilba Lima Souza, ${ }^{1}$ Ana Katherine Gonçalves (D) , Maria de Lourdes Costa Silva (1) ${ }^{2}$

To cite: Oliveira DJD, Medeiros KS, Sarmento ACA, et al. Use of glucose for pain management in premature neonates: a systematic review and meta-analysis protocol. BMJ Open 2021;11:e052901. doi:10.1136/ bmjopen-2021-052901

- Prepublication history and additional supplemental material for this paper are available online. To view these files, please visit the journal online (http://dx.doi.org/10.1136/ bmjopen-2021-052901).

Received 27 April 2021 Accepted 24 September 2021

Check for updates

(c) Author(s) (or their employer(s)) 2021. Re-use permitted under CC BY-NC. No commercial re-use. See rights and permissions. Published by BMJ.

${ }^{1}$ Universidade Federal do Rio Grande do Norte, Natal, Brazil ${ }^{2}$ Nursing Department, Universidade Federal do Rio Grande do Norte, Natal, Brazil

Correspondence to Professor Maria de Lourdes Costa Silva;

lurdinhafoc@hotmail.com

\section{ABSTRACT}

Introduction Therapeutic management of neonatal pain is essential to reduce changes in initial and subsequent development. Although glucose has been shown to be effective in relieving pain, concentrations and dosages remain to be standardised. The objective of this systematic review and meta-analysis is to identify the efficacy of glucose as an analgesic in preterm infants.

Methods and analysis The Web of Science, Science Direct, Scopus, Cochrane Central Register of Controlled Trials, ClinicalTrials.gov, PubMed, Medline, Latin American and Caribbean Health Sciences Literature and Embase databases will be researched for randomised studies published until December 2021. This systematic review and meta-analysis will include studies investigating the use of glucose for pain control in premature neonates. The primary outcome will be pain relief. Three independent reviewers will select the studies and extract the data from original publications. The risk of bias was assessed using the Cochrane risk of bias tool. Data synthesis will be performed using the Review Manager software (RevMan V.5.2.3). We will evaluate heterogeneity based on $\mathrm{I}^{2}$ statistics. In addition, quantitative synthesis will be performed if the included studies are sufficiently homogeneous.

Ethics and dissemination Ethical approval for the research will not be required for this systematic review. The results of this study will be published in an international journal.

Trial registration number This protocol was submitted to the International Prospective Register of Systematic Reviews (PROSPER0, number CRD42021236217).

\section{INTRODUCTION}

\section{Description of the condition}

Neonatal pain is an extremely important component in the therapeutic management of children. Over the years this issue has been the focus of research, our understanding of pain management in neonatal care has undergone several transformations. Though previously considered a non-existent element, the concept of pain management as an integral part of neonatal care has gained attention. ${ }^{1}$
Strengths and limitations of this study

- Three reviewers will independently select eligible studies, extract data and evaluate the risk of bias.

- In case of unavailable full texts, we will contact the corresponding authors to access the study.

- No establishing language restrictions will be used.

- The data may not be available to allow a meta-analysis.

The challenges of adaptation to extrauterine life may lead to the requirement of care for newborns in neonatal intensive care or intermediate care units. These environments provide treatments to improve clinical evolution. However, many of these measures involve painful procedures, and babies are exposed to an estimated 7-17 potentially painful procedures daily. ${ }^{2}$ In addition, stress related to neonatal pain in prematurely born children is associated with significant early and later developmental changes such as postnatal growth retardation, a deficit in early neurodevelopment, exacerbated cortical activation and altered brain development. ${ }^{3}$

Cumulative exposure to pain has the potential to impair brain development in preterm infants, ${ }^{4}$ and the exposure of preterm neonates to stress has been related to epigenetic modulation. Therefore, effective and safe strategies are needed for the treatment of neonatal pain through the use of sweet solutions. Considering that new clinical research is constantly being conducted internationally, it is an opportune time to carry out periodically updated analyses to verify the findings. ${ }^{5}$

Description and mechanism of the intervention Pharmacological therapies such as sweet solutions for the relief of mild to moderate pain, as well as non-pharmacological measures such as non-nutritive sucking, breast feeding, 
skin-to-skin contact and facilitated containment have been effective as rated by the American Academy of Pediatrics $2016 .^{6-8}$

Although sucrose solution is used internationally to study the behavioural effects in the control of neonatal pain, its effect on brain activity after a painful procedure remains uncertain. ${ }^{9}$ Therefore, it is extremely important to investigate the analgesic effects of alternative solutions such as glucose. ${ }^{10}$ Although there is no scientific consensus between dose and concentration, it was estimated that 20\%-30\% glucose would be sufficient for pain relief in neonates. ${ }^{11} 12$ However, it is still necessary to investigate and determine safe and effective standard doses and concentrations.

The mechanism of analgesia induced by sweet solutions has not been fully elucidated. While animal studies provided more convincing evidence of the involvement of the endogenous opioid system, human studies are ambiguous. Thus, to date, evidence supports the theory that analgesia induction mechanism can be mediated by opioid and non-opioid pathways. ${ }^{13}$

\section{Significance of this review}

The administration of a minimal effective concentration and dosage for procedural pain relief is crucial for noniatrogenic pain treatment. ${ }^{911-16}$ In this sense, it is worth mentioning that neonatal pain has been undertreated in health services. ${ }^{1}$ Neonates need adequate treatment for procedural pain management. ${ }^{1}{ }^{11-16}$ Therefore, studies with concrete and reliable scientific data are desirable to facilitate changes in institutional protocols for pain management.

In addition, inadequate management of procedural pain may lead to decreased transcutaneous oxygen saturation, increased heart and respiratory rates, and increased cortisol levels in children, ${ }^{1}$ as well as other more severe developmental changes such as postnatal growth retardation, early neurodevelopmental deficits, exacerbated cortical activation and altered brain development. ${ }^{3}$

Considering the range of effects observed with inadequate neonatal pain management in premature infants, ${ }^{2-5}$ there is an urgent need to investigate safe and effective alternatives for this population.

\section{Objectives}

The objective of this systematic review protocol and metaanalysis is to identify the efficacy of glucose as an analgesic intervention in preterm infants.

\section{METHODS AND ANALYSIS}

This protocol was submitted and approved by the International Prospective Register of Systematic Reviews (PROSPERO, number CRD42021236217). The Preferred Reporting Items for Systematic Reviews and Meta-Analyses (PRISMA) ${ }^{17}$ are used to design this protocol.

\section{Eligibility criteria}

Studies that meet the following criteria will be included: randomised clinical trials conducted with premature neonates that evaluate glucose concentrations and/or doses in the management of neonatal procedural pain. There were no language restrictions when selecting the studies.

Studies with neonatal patients in the immediate postoperative period, babies with neurological disorders, sedated patients and non-validated pain assessment scales will be excluded.

Patients, intervention, comparisons, results and type of study Patients: Premature newborns (human babies born before 37 weeks of gestation), ${ }^{18}$ hospitalised in the neonatal intensive care unit requiring painful therapeutic/diagnostic procedures and, consequently, therapeutic management of pain.

- Late premature: Newborn with 32 to less than 37 weeks of gestational age. ${ }^{18}$

- Very premature: Newborn with 28 weeks to less than 32 weeks of gestational age. ${ }^{18}$

- Extreme premature: Newborn under 28 weeks of gestational age. $^{18}$

Intervention: Oral administration of glucose for the management of procedural pain. ${ }^{19}$

\section{Comparison/control}

- Absence of therapy for pain management ${ }^{720}$

- Placebo

- Different glucose doses/concentrations ${ }^{720}$

- Sucrose/sweet solutions ${ }^{710}$

- Music therapy ${ }^{21}$

- The Kangaroo method ${ }^{22}$

- Non-nutritive sucking ${ }^{18}$

- Breast milk ${ }^{23}$

- Breast feeding ${ }^{23}$

- Facilitated tucking. ${ }^{20}$

Primary outcome

Pain assessed by validated pain measurement tools.

- Neonatal Facial Coding System ${ }^{3}$

- Premature Infant Pain Profile $e^{13}$

- Premature Infant Pain Profile - Revised ${ }^{13}$

- Neonatal Pain and Sedation Scale ${ }^{11}$

- Neonatal Infant Pain Scale ${ }^{12}$

- Behavioral Indicators of Infant Pain ${ }^{12}$

In addition to the aforementioned neonatal pain assessment scales, there were no limitations on assessment instruments, as long as they are cited in primary studies.

Secondary outcome: Infant heart rate, oxygen saturation, crying time, and newborn salivary cortisol level ${ }^{24}$ and neurophysiological assessment. ${ }^{25} 26$

Type of study: This systematic review and meta-analysis will include randomised clinical trials.

Involvement of the patients and the public

Patients and the public will not be involved.

\section{Search strategy}

The search strategies in this study will be applied to the Web of Science, Science Direct, Scopus, Cochrane Central 


\begin{tabular}{cl}
\hline Table 1 & Search strategy \\
\hline 1 & Newborn \\
\hline 2 & Infant \\
\hline 4 & Premature \\
\hline 5 & Extremely premature \\
6 & Neonate \\
\hline 7 & Glucose \\
\hline 8 & Sweet solutions \\
\hline 9 & Sweet-tasting solution \\
\hline 10 & Music therapy \\
\hline 11 & Kangaroo-mother care method \\
\hline 12 & Breast milk \\
\hline 13 & Breastfeeding \\
\hline 14 & Sucking behavior \\
\hline 15 & Pain management \\
\hline 16 & Analgesia \\
\hline 17 & Randomized clinical trial \\
\hline
\end{tabular}

Register of Controlled Trials, ClinicalTrials.gov, PubMed/ Medline, Latin American and Caribbean Health Sciences Literature, and Embase databases to search for articles published until December 2021. Search strategies for each database are listed in the supplementary file.

An initial limit in the research period is not included. Thus, we will search for studies published until December 2021. Furthermore, grey literature will be searched using appropriate databases (eg, OpenGrey) and we will analyse the reference lists, as appropriate, in terms of studies that contribute to the composition of the sample corpus. To expand the content that will compose the sample corpus of this work, there will be no language restrictions.

The descriptors used in data search will include the following: (newborn OR infant OR extremely premature OR neonate) AND (glucose OR sweet solutions OR sweettasting solution) AND (music therapy OR kangaroomother care method OR breast milk OR breastfeeding OR sucking behavior OR facilitated tucking) AND (pain management OR analgesia) AND (randomized clinical trial) (table 1). Eligible studies will also be selected from the reference lists of the recovered articles.

\section{Data collection and analysis}

Selection of studies

Three authors (DJDO, KSM and MLCS) will independently track the search results according to the review search terms. Duplicate studies and reviews will be excluded. Two reviewers, DJDO and ACAS, will then review the full text to determine if the studies meet the inclusion criteria. Discrepancies will be resolved by a third reviewer (KSM). The selection of studies is summarised in the PRISMA ${ }^{18}$ flow chart (figure 1).

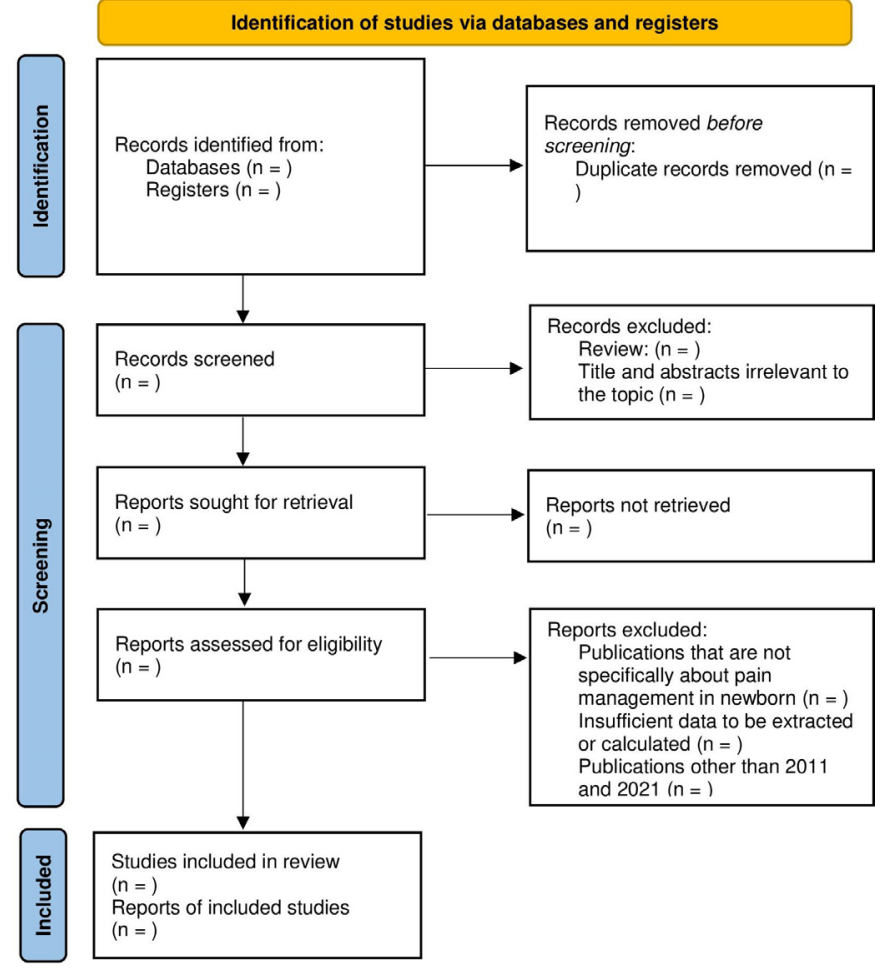

Figure 1 Flow diagram of the search for eligible studies on oral glucose in pain management: CENTRAL, Cochrane Central Register of Controlled Trials.

For the selection of studies, we will use Rayyan, an intelligent online tool for screening papers in systematic reviews. ${ }^{27}$

\section{Data extraction and management}

The data will be extracted by DJDO, KSM and MLCS. The extracted characteristics will include the surnames of the first authors, year of publication, study location (country), study design, primary objective, population, sample size, period and follow-up, inclusion/exclusion criteria, doses and/or glucose concentrations used, controls, scales used, biological variables (average days of life, gestational age, corrected gestational age, Appearance, Pulse, Grimace, Activity, and Respiration Index, basic pathologies) and primary results.

Standardised data extraction forms will be created for this review, and the results will subsequently be entered into a database. All data entries will be double-checked.

\section{Addressing lost data}

In case of missing data, the authors will attempt to contact the first authors, coauthors or corresponding authors of the article by phone, email or post. If the necessary information cannot be obtained, the data will be excluded from the analysis and will be addressed in the discussion section.

\section{Bias risk assessment}

Two authors, DJDO and MLCS, will independently assess the risk of bias in eligible studies using Cochrane's Risk of Bias tool. ${ }^{28}$ The risk of bias will be categorised as high, 
low or unclear for individual elements among the items listed in the five domains (selection, performance, friction, reporting and others).

\section{Heterogeneity assessment}

The heterogeneity among the study results will be evaluated using standard $\chi^{2}$ tests with a significance level of $\mathrm{p}<0.1$. The $\mathrm{I}^{2}$ values, which are a quantitative measure of inconsistency between studies, will be calculated, with values of $0 \%$ and $\geq 50 \%$ indicating no heterogeneity and considerable levels of heterogeneity, respectively. However, heterogeneity will be assessed only if it is appropriate to conduct a meta-analysis.

\section{Analysis}

The data will be analysed using Review Manager software (RevMan V.5.2.3). For dichotomous results, the ORs and 95\% CIs will be extracted/calculated from each study. In case of heterogeneity $\left(\mathrm{I}^{2} \geq 50 \%\right)$, random-effects models will be used to combine the studies to calculate the ORs and 95\% CIs using the DerSimonian-Laird algorithm, which provides functions to conduct meta-analyses in R. For meta-analysis of continuous data, the mean difference and the standardised mean difference will be realised.

Other features and results of the study will be summarised in a table, in case the meta-analysis cannot include all or some of the studies. Sensitivity analyses will be used to explore the robustness of the conclusions regarding study quality and sample sizes, which will be possible only if the proposed meta-analysis of the studies is feasible. The results of sensitivity analysis will be summarised in a table.

\section{Evaluation of the quality of the evidence}

To classify the strength of the evidence from the included data, the Grading of Recommendations Assessment, Development and Evaluation approach will be used. The summary of the entire evaluation will be incorporated into the broader measurements to allow the assessment of bias risk, consistency, candour and accuracy. ${ }^{29}$

\section{Ethics and dissemination}

Considering the use of previously published data, ethical approval of the research is not required for this systematic review. The results of this study will be published in an international journal. If new and robust evidence becomes available, the results will be updated.

\section{DISCUSSION}

In this systematic review and meta-analysis, we aim to evaluate the efficacy of glucose as an analgesic intervention in preterm infants. Exposure to pain negatively affects child development, with short-term and long-term effects, such as prolonged and chronic pain, as well as changes in neurobehavioural responses to future painful episodes. ${ }^{24}$ For children born prematurely, pain-related stress is associated with significant changes in early and later development, such as postnatal growth retardation, deficits in early neurodevelopment, exacerbated cortical activation and altered brain development. ${ }^{3}$

The cortical effect of sucrose on pain control remains controversial. A recentstudyshowed that, although it reduced acute pain behaviour in babies, the sucrose did not induce cerebral effects. ${ }^{9}$ Glucose, regardless of its association with maternal restraint, has been shown to help block or weaken the processing of cortical pain in neonates. ${ }^{12}$ However, it is necessary to accurately determine the minimum dose and concentration to avoid iatrogenic events.

Discussion on the adverse events that occur in the long term is still incipient in the literature. Regarding short-term events, there is mention of minor events (such as choking and suffocation) that occur in different therapies, including glucose. ${ }^{15}$ We expect that our study will provide accurate data about the efficacy of glucose as an analgesic intervention in preterm infants, in addition to determining sufficient concentration and dosage for neonatal analgesia, contributing to safe and effective clinical practices.

Acknowledgements The authors thank the Ministry of Education/Brazil for providing scholarship for the postgraduate course in neonatal intensive care, in the modality of Multiprofessional Residence, which provided the opportunity to develop and perform this research. The authors also thank the Federal University of Rio Grande do Norte (UFRN) for this opportunity.

Contributors AKG, ACAS, APFC and FJDO contributed to the design of this review. DJDO, KSM and MLCS drafted the protocol manuscript. ACAS and KSM revised the protocol manuscript. DJDO, KSM and MLCS developed and implemented the search strategies. DJDO, KSM, and MLCS will track potential studies, extract data and assess quality. In cases of disagreement between the data extractors, NLS will advise on the methodology and act as a referee. DJD0 will complete the data synthesis. All authors approved the final version for publication.

Funding The authors have not declared a specific grant for this research from any funding agency in the public, commercial or not-for-profit sectors.

Competing interests None declared.

Patient consent for publication Not applicable.

Provenance and peer review Not commissioned; externally peer reviewed. Not Applicable.

Supplemental material This content has been supplied by the author(s). It has not been vetted by BMJ Publishing Group Limited (BMJ) and may not have been peer-reviewed. Any opinions or recommendations discussed are solely those of the author(s) and are not endorsed by BMJ. BMJ disclaims all liability and responsibility arising from any reliance placed on the content. Where the content includes any translated material, BMJ does not warrant the accuracy and reliability of the translations (including but not limited to local regulations, clinical guidelines, terminology, drug names and drug dosages), and is not responsible for any error and/or omissions arising from translation and adaptation or otherwise.

Open access This is an open access article distributed in accordance with the Creative Commons Attribution Non Commercial (CC BY-NC 4.0) license, which permits others to distribute, remix, adapt, build upon this work non-commercially, and license their derivative works on different terms, provided the original work is properly cited, appropriate credit is given, any changes made indicated, and the use is non-commercial. See: http://creativecommons.org/licenses/by-nc/4.0/.

ORCID iDs

Ana Katherine Gonçalves http://orcid.org/0000-0002-8351-5119

Maria de Lourdes Costa Silva http://orcid.org/0000-0001-7251-8966

\section{REFERENCES}

1 Johnston C. Neonatal pain: a journey spanning three decades. Paediatric and Neo Pain 2020;2:33-9. 
2 Cruz MD, Fernandes AM, Oliveira CR. Epidemiology of painful procedures performed in neonates: a systematic review of observational studies. Eur J Pain 2016;20:489-98.

3 Valeri BO, Holsti L, Linhares MBM. Neonatal pain and developmenta outcomes in children born preterm: a systematic review. Clin J Pain 2015;31:355-62.

4 Boggini T, Pozzoli S, Schiavolin P, et al. Cumulative procedural pain and brain development in very preterm infants: a systematic review of clinical and preclinical studies. Neurosci Biobehav Rev 2021:123:320-36.

5 Casavant SG, Cong X, Moore J, et al. Associations between preterm infant stress, epigenetic alteration, telomere length and neurodevelopmental outcomes: a systematic review. Early Hum Dev 2019;131:63-74.

6 Harrison D, Larocque C, Bueno M, et al. Sweet solutions to reduce procedural pain in neonates: a meta-analysis. Pediatrics 2017;139. doi:10.1542/peds.2016-0955. [Epub ahead of print: 1612 2016].

7 Bueno M, Yamada J, Harrison D, et al. A systematic review and meta-analyses of nonsucrose sweet solutions for pain relief in neonates. Pain Res Manag 2013;18:153-61.

8 Committee on Fetus and Newborn and Section on Anesthesiology and Pain Medicine. Prevention and management of procedural pain in the neonate: an update. Pediatrics 2016;137:e20154271.

9 Slater R, Worley A, Fabrizi L, et al. Evoked potentials generated by noxious stimulation in the human infant brain. Eur $J$ Pain 2010;14:321-6.

10 Stevens B, Yamada J, Ohlsson A, et al. Sucrose for analgesia in newborn infants undergoing painful procedures. Cochrane Database Syst Rev 2016;7:CD001069.

11 Hillman BA, Tabrizi MN, Gauda EB, et al. The neonatal pain, agitation and sedation scale and the bedside nurse's assessment of neonates. J Perinatol 2015;35:128-31.

12 Bembich S, Cont G, Causin E, et al. Infant analgesia with a combination of breast milk, glucose, or maternal holding. Pediatrics 2018;142:e20173416.

13 Eriksson M, Finnström O. Can daily repeated doses of orally administered glucose induce tolerance when given for neonatal pain relief? Acta Paediatr 2004;93:246-9.

14 American Academy of Pediatrics, Canadian Pediatrics Society. Prevention and management of pain in neonate: an update. American Academy of Pediatrics; 2006: 118.

15 Stevens B, Yamada J, Campbell-Yeo M, et al. The minimally effective dose of sucrose for procedural pain relief in neonates: a randomized controlled trial. BMC Pediatr 2018;18:85.
16 Lima AGCF, Santos VS, Nunes MS, et al. Glucose solution is more effective in relieving pain in neonates than non-nutritive sucking: a randomized clinical trial. Eur J Pain 2017;21:159-65.

17 Moher D, Liberati A, Tetzlaff J, et al. Preferred reporting items for systematic reviews and meta-analyses: the PRISMA statement. PLoS Med 2009;6:e1000097.

18 Quinn J-A, Munoz FM, Gonik B, et al. Preterm birth: Case definition \& guidelines for data collection, analysis, and presentation of immunisation safety data. Vaccine 2016;34:6047-56.

19 Kumari S, Datta V, Rehan H. Comparison of the Efficacy of Oral $25 \%$ Glucose with Oral 24\% Sucrose for Pain Relief during Heel Lance in Preterm Neonates: A Double Blind Randomized Controlled Trial. J Trop Pediatr 2017;63:30-5.

20 Cignacco EL, Sellam G, Stoffel L, et al. Oral sucrose and "facilitated tucking" for repeated pain relief in preterms: a randomized controlled trial. Pediatrics 2012;129:299-308.

21 van Dokkum NH, Jaschke AC, Ravensbergen A-G, et al. Feasibility of live-performed music therapy for extremely and very preterm infants in a tertiary NICU. Front Pediatr 2020;8:581372.

22 Boundy EO, Dastjerdi R, Spiegelman D, et al. Kangaroo mother care and neonatal outcomes: a meta-analysis. Pediatrics 2016;137. doi:10.1542/peds.2015-2238. [Epub ahead of print: 2312 2015].

23 Shah PS, Herbozo C, Aliwalas LL, et al. Breastfeeding or breast milk for procedural pain in neonates. Cochrane Database Syst Rev 2012;12:CD004950.

24 Brummelte S, Chau CMY, Cepeda IL, et al. Cortisol levels in former preterm children at school age are predicted by neonatal procedural pain-related stress. Psychoneuroendocrinology 2015;51:151-63.

25 Hartley C, Slater R. Neurophysiological measures of nociceptive brain activity in the newborn infant--the next steps. Acta Paediatr 2014;103:238-42.

26 Benoit B, Martin-Misener R, Newman A, et al. Neurophysiological assessment of acute pain in infants: a scoping review of research methods. Acta Paediatr 2017;106:1053-66.

27 Ouzzani M, Hammady H, Fedorowicz Z, et al. Rayyan-a web and mobile APP for systematic reviews. Syst Rev 2016;5:210.

28 Higgins JPT, Altman DG, Gøtzsche PC, et al. The Cochrane collaboration's tool for assessing risk of bias in randomised trials. BMJ 2011;343:d5928.

29 Balshem H, Helfand M, Schünemann HJ, et al. Grade guidelines: 3. rating the quality of evidence. J Clin Epidemiol 2011;64:401-6. 